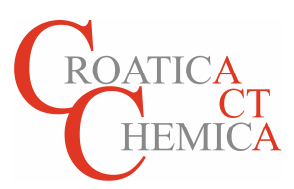

\title{
Contemporary Diffraction Methods in Study of Polycrystals
}

\author{
Stanko Popović, ${ }^{*}$ Antun Tonejc, Željko Skoko
}

\author{
Department of Physics, Faculty of Science, University of Zagreb, Bijenička cesta 32, 10000 Zagreb, Croatia \\ * Corresponding author's e-mail address: spopovic@phy.hr
}

RECEIVED: September 29, 2015 * REVISED: December 2, 2015 * ACCEPTED: December 2, 2015

THIS PAPER IS DEDICATED TO DR. SVETOZAR MUSiĆ ON THE OCCASION OF HIS 70Th BIRTHDAY

\begin{abstract}
Diffraction in the polycrystal/crystalline powder is one of the most powerful techniques in study of microstructure and crystal structure of solids. This technique enables, in synergy with microscopic, spectroscopic and other physical techniques, a complete analysis of onephase and multi-phase substances, that are important in scientific and technological fields. Information on microstructure and crystal structure of a substance is stored in its diffraction pattern; in order to reveal this information, the diffraction pattern should be decoded by application of adequate mathematical and physical procedures which may often be rather complex. During the last decades, diffraction techniques in the polycrystal are developing rapidly due to the introduction of sophisticated instrumentation, powerful computers and by application of synchrotron radiation. This advance enables the collection and interpretation of diffraction data in a short real time as well as the study of time-resolved dynamic processes in the crystalline substance. Possibilities of diffraction techniques in the polycrystal are concisely described and illustrated by examples of authors' scientific studies.
\end{abstract}

Keywords: X-ray diffraction, microstructure, crystal structure, recent advancement in diffraction.

\section{DIFFRACTION IN THE POLYCRYSTAL}

D IFFRACTION is a phenomenon in which the beam of $X$-rays, electrons or neutrons, by passing through a single crystal or a polycrystal, deviates from the initial direction due to the scattering on the structural motifs (atoms, ions, molecules) in the crystal. The scattered waves give rise to diffraction maxima in a set of discrete space directions due to their mutual constructive interference. These directions strongly depend on the periodicity and symmetry of the crystal. The set of diffraction maxima represent the diffraction pattern of the single crystal/polycrystal. The intensities of diffraction maxima depend on the nature of structural motifs and their mutual space arrangement. Therefore, diffraction techniques are ideal in the study of the crystal structure and microstructure: space periodicity in the crystal, unit-cell parameters (unit-cell edges $a, b$ and $c$ and angles among them, $\alpha, \beta$ and $\gamma$ ), symmetry elements which determine the space group, space periodical arrangement of structural motifs, identification of particular atoms and their coordinates, definition of the nature and length of their mutual chemical bonds, the angles among the chemical bonds, the size and shape of crystallites, distribution of crystallite sizes, the nature of the crystallite/grain boundary, stresses and strains inside the crystallite, point, planar and volume defects inside the crystallite, phase transitions in a given substance and the definition of its phase diagram, etc.

In determination of the crystal structure, diffraction in the single crystal has an advantage in relation to the diffraction in the polycrystal. That is a consequence of the fact that the 3D data, obtained by diffraction in the single crystal, are transferred (condensed) in the 1D data as obtained by diffraction in the polycrystal. However, diffraction in the polycrystal possesses a series of advantages in relation to that in the single crystal, and is one of the most powerful techniques in the study of solids. Diffraction in the polycrystal enables, beside a simple preparation of the specimen, fast and non-destructive analysis of one-phase and multiphase substances important in the solid state physics and chemistry, biology, mineralogy, archaeology, medicine, 
forensics and in many technologies, metallurgy, pharmaceutics, semiconductor and cement industries.

A crystalline substance is not always available as a single crystal which possesses a quality and size suitable for determination of the crystal structure by diffraction techniques. In the majority of such cases the crystal structure and microstructure can be studied by contemporary diffraction techniques using the polycrystal/crystalline powder. There are many excellent textbooks describing in detail these techniques. ${ }^{[1-4]}$ The technique was introduced by Peter Debye and Paul Scherrer in 1916 in Göttingen ${ }^{[5]}$ and, independently of them, by Albert Hull in 1917 in Schenectady (USA). ${ }^{[6]}$ It is important to state that Toahiko Terada in Tokyo studied singe crystals and polycrystals in 1913, according to the paper by Nishikawa ${ }^{[7]}$ appearing in the monographs Fifty Years of X-ray Diffraction (1962). ${ }^{[8]}$

A simple interpretation of diffraction in the polycrystal (applicable also for diffraction in the single crystal) was formulated by William Lawrence Bragg. ${ }^{[9]}$ A particular diffraction maximum originates by reflexion of the incident beam of X-rays, electrons or neutrons from a given set of mutually parallel and equidistant crystal lattice planes. A diffraction maximum, denoted by three integers, $h k l$, belonging to the crystal lattice planes $(h k l)$ (where $h, k$ and $I$ being Miller indices), having the interplanar spacing $d_{h k l}$, appears only at that (Bragg) angle, $\Theta_{h k l}$, between the incident beam and the set of the crystal lattice planes for which the path difference between the rays reflected from the neighbouring planes is equal to the whole number of their wavelength (the Bragg law):

$$
2 d_{h k l} \sin \Theta_{h k l}=n \lambda
$$

A polycrystalline sample, which is suitable to be studied, should contain a great number of randomly oriented crystal grains / crystallites, sufficiently small that all orientations (of their crystal axes) are about equally present in the specimen volume irradiated by the incident beam. If that condition is fulfilled, then approximately an equal number of crystallites contribute to a given diffraction maximum. That number is a small fraction of the total number of crystallites in the specimen, but it should be sufficiently great that the diffraction maximum could be recorded with a satisfactory accuracy. It is accepted in the literature that a relatively well defined and sharp diffraction maximum can be obtained if the grains/crystallites in the specimen are smaller than $\sim 10 \mu \mathrm{m}$ and larger than $\sim 200 \mathrm{~nm}$.

$X$-rays diffracted in the polycrystal form a set of cones which have a common axis coinciding with the incident beam. Each diffraction cone (i.e. diffraction maximum) with the opening of $4 \Theta_{h k l}$, originates by the reflexion from all sets of crystal lattice planes (one set in one crystallite), having the same Miller indices, $(h k l)$, and therefore the same interplanar spacing, $d_{h k l}$, which satisfies the Bragg law. The classic instrument for obtaining the X-ray diffraction pattern of the polycrystal is the Debye-Scherrer camera. That camera is loaded by a cylindrical film which adheres to the inner wall of the camera. The camera contains: a collimator with a filter for producing an approximately monochromatic incident beam having a small crosssection and being normal to the film axis; a goniometer head which serves as the specimen holder enabling the placement of the specimen into the incident beam. The cylindrical specimen, having the diameter of $\approx 0.3 \mathrm{~mm}$, coincides with the film axis and may rotate about it. The diffraction pattern on the film consists of a set of diffraction lines as cross-sections of diffraction cones with the film.

The diffraction pattern of the polycrystal exhibiting narrow and well-resolved diffraction lines can be achieved by the Guinier camera in which both the thin specimen (having the surface of $\approx 1 \mathrm{~cm}^{2}$, and the cylindrical film or the $2 \mathrm{D}$ detector adhere to the inner wall of the camera. The specimen is exposed to a divergent X-ray beam which, by passing through the specimen, is divided in a set of diffracted beams converging toward the film or the $2 \mathrm{D}$ detector.

A modern highly sofisticated electronic instrument for obtaining the diffraction pattern of the polycrystal/crystalline powder, is the counter diffractometer. It contains: the source of X-rays produced by a DC high-voltage; goniometer for the automatic change of the orientation of the flat specimen in relation to the incident beam of X-rays; monochromator and $1 \mathrm{D}$ detector or the position-sensitive detector; additional electronic instruments and a powerful computer having installed the appropriate software for the collection and interpretation of the diffraction data (diffraction pattern) in terms of the crystal structure and microstructure. The specimen is in the form of a thin cylinder/a thin square (having the diameter/the edge of $\approx 1 \mathrm{~cm}$ ) which may be a compact polycrystal or a crystalline powder fixed by a glue. The goniometer axis is tangent to the specimen surface. The separation between the $\mathrm{X}$-ray source and the goniometer axis is equal to the separation between the goniometer axis and the receiving slit of the detector. For the diffractometer having the Bragg-Brentano geometry, the angle between the incident beam and the specimen surface is always equal to the angle between the specimen surface and the direction toward the receiving slit of the detector. The equality of these angles during the collection of the diffraction pattern is maintained in such a way that the angular speed of the detector about the goniometer axis is twice that of the specimen surface. Any time when that angle satisfies the Bragg law, the detector records a diffraction maximum (diffraction line); the detector scans the diffraction 
line any time when the receiving slit passes through one of the diffraction cones. In such a way the detector scans positions, intensities and profiles of diffraction lines which are interpreted in terms of the crystal structure and microstructure. All the information on the crystal structure and microstructure is stored in the generated diffraction pattern; that information can be revealed by the appropriate mathematical and physical procedures, which may be rather complex. The diffractometer may have installed attachments for study of the behaviour of the substance at low and high temperature and at high pressure.

\section{INFORMATION REVEALED BY DIFFRACTION IN POLYCRYSTALS}

The diffraction pattern of a given crystalline substance may not contain diffraction maxima for certain sets of crystal lattice planes due to the special groups of symmetry elements in the crystal structure. These systematic absences enable the determination of the space group of the substance chemical element or chemical compound. Each chemical element/chemical compound has its characteristic set of interplanar spacings and its own crystal structure; therefore, it has its own diffraction pattern. The application of diffraction techniques, and especially diffraction of X-rays, in the polycrystal thus enables the identification of the studied crystalline substance. This task may be much helped and simplified by comparison of the diffraction pattern of the studied substance with the diffraction and structural data stored in the Powder Diffraction File, International Centre for Diffraction Data, ${ }^{[10]}$ or in the Cambridge Structural Database, Cambridge Crystallographic Data Centre. The ICDD recently has launched a new database, PDF-4. Its purpose is to provide the analysis tools based on the combination of single crystal and powder reference data, housed in a database format that could provide flexibility and useful data mining. PDF-4 contains both amorphous and nanomaterial references. The use of digital patterns for both the substance being analyzed and the reference substance enables to study e.g. the crystallite size, crystallite orientation and instrumental/specimen contribution to the coherent and incoherent scattering and the analysis of crystallinity. Digital simulation tools are radiation independent so that one can use X-ray, neutron, electron or synchrotron diffraction data. The calculated intensity scaling factors, $I / I_{c},{ }^{[11]}$ for both $\mathrm{X}$-rays and neutron analyses are added. ${ }^{10]}$

The interplanar spacing of the set of crystal lattice planes $(h k l)$ is given by the equation

$$
\frac{1}{d_{h k l}}=\frac{|h \mathbf{b} \times \mathbf{c}+k \mathbf{c} \times \mathbf{a}+| \mathbf{a} \times \mathbf{b} \mid}{\mathbf{a}(\mathbf{b} \times \mathbf{c})} .
$$

This equation reduces to a simpler form for crystal systems of a higher symmetry, e.g. to $\left(1 / d_{h k l}\right)^{2}=a^{-2}\left(h^{2}+k^{2}+R^{2}\right)$ for the cubic system and to $\left(1 / d_{h k l}\right)^{2}=a^{-2} h^{2}+b^{-2} k^{2}+c^{-2} p^{2}$ for the orthorhombic system. One of the first tasks in the interpretation of the diffraction pattern is to find out the unique link between a given diffraction maximum and the corresponding set of crystal lattice planes, i.e. to determine the Miller indices. The Bragg angles of diffraction maxima are given in the diffraction pattern itself; then the unit-cell parameters can be calculated according to Eqs. (1) and (2). The determination of the Miller indices is simple for highsymmetry crystal systems. For low-symmetry crystal systems that task is not always straightforward - there is no software which would always give unique solutions, especially in the case of a big unit cell.

A great advantage of diffraction techniques with polycrystals is manifested in study of the mixtures of chemical elements and/or chemical compounds. Each phase of the multi-phase sample gives its own diffraction pattern independently of other phases present in the sample. The resulting diffraction pattern is the superposition of diffraction patterns of particular phases in the sample. That fact enables the quantitative phase analysis, that is, the determination of mass/weight/molar fractions of the phases present in the mixture. Details of the procedures of the quantitative phase analysis are given in the literature. ${ }^{[1,11,12]}$

An amorphous substance does not exhibit sharp diffraction maxima; it is recognized according to a very broad maximum, or two or three maxima, due to the X-rays scattered in the substance. The patterns of the scattered X-rays are similar for different amorphous substances; thus, the identification of the amorphous substance is not possible on the basis of such a pattern. However, by means of the Fourier transform of the angular dependence of the scattered $\mathrm{X}$-rays it is possible to find the space distribution of the electron density in the vicinity of a given atom/ion, e.g. in metallic glasses. If there are two kinds of atoms, e.g. a metal oxide, it is not possible to distinguish the environment of the metal atom from that of the oygen atom. ${ }^{[3]}$ It should be mentioned that the diffraction pattern of the studied substance, having the grains/crystallites smaller than $\sim 5 \mathrm{~nm}$, tends to become similar to the pattern of an amorphous substance; then complementary experimental techniques should be used, e.g. high-resolution transmission electron microscopy, in order to reveal the real structure/microstructure of the studied substance.

Important information on the crystalline substance can be determined from small shifts and changes of intensities of diffraction maxima, due to a change of the chemical composition, temperature and pressure. This point is very essential in the study of solid solutions, as well as the 
natural samples, minerals. The solid solution is a statistically homogeneous space distribution of atoms/ions of a chemical element/chemical compound (solute) solved in the crystal structure of another chemical element/chemical compound (solvent). The atoms/ions of the solute can replace atoms/ions of the solvent in positions defined by the solvent space group (substitutional atoms/ions) or can be located in positions which are not defined by the solvent space group (interstitial atoms/ions). In case the atomic/ionic radii of the two sorts of atoms/ions in the substitutional solid solution are different, angular shifts of the solvent diffraction maxima take place and these shifts depend on the Bragg angle. For the interstitial solid solution an increase of the unit-cell edges is observed (i.e. a shift of diffraction maxima towards smaller Bragg angles); these changes are usually anisotropic except for the cubic system. The shifts of diffraction maxima yield the concentration of the solute and the solubility limit. A change of temperature of the substance also causes shifts of diffraction maxima; this enables determination of the thermal expansion coefficient and its anisotropy caused by the nature of the chemical bonds along the crystal axes. Therefore, the modern diffractometer provides information on the change of the crystal structure and microstructure of the substance during the change of its chemical composition, temperature and pressure, this being very important in definition of the phase diagram of the substance. Diffraction techniques are unique in studies of the solid state and cannot be replaced by other techniques. ${ }^{[13,14]}$

In the choice of diffraction maxima, especially in study of microstructure, one has to keep in mind the fact that the shift of the diffraction maximum, $\Delta \Theta_{h k l}$, due to a change of the interplanar spacing, $\Delta d_{h k l} / d_{h k l}$, is proportional to $\tan \Theta_{h k l}$, as it can be derived from the Bragg law:

$$
\Delta \Theta_{h k l}=-\tan \Theta_{h k l} \frac{\Delta d_{h k l}}{d_{h k l}} .
$$

According to (3), the shift of the diffraction maximum due to a change of the interplanar spacing increases rapidly with the Bragg angle. In the accurate measurement of the dependence of the unit-cell parameters on the temperature and pressure, in the study of solid solutions and solubility limits, phase transitions etc., it is recommended to follow the angular positions of diffraction maxima, having uniquely determined Miller indices, at as high as possible Bragg angles. ${ }^{[1,3,15]}$

Many chemical elements and compounds undergo phase transitions due to a change of the temperature and pressure. In the phase transition, atoms/ions/molecules change their mutual space arrangement to a more stable than the previous one. The phase transition is easily recognized in the diffraction pattern. In accordance with the relation among the symmetry elements of the previous and the new phase, these phenomena may take place: the appearance of new diffraction maxima and disappearance of the previous maxima, splitting of a diffraction maximum in two or more new maxima, or in merging two or more maxima into a single maximum, or an abrupt change of the Bragg angle of diffraction maxima due to an abrupt change of the unit-cell parameters and/or the symmetry of the structure, which is accompanied by the change in physical properties, e.g. thermal expansion coefficient. ${ }^{[13,14]}$

The crystallite grains/crystallites may be preferentially oriented in the polycrystalline sample, due to cold work (metals), pressing (layered structures, plastics) etc. In that case, the intensities of diffraction maxima are changed. By means of adequate diffraction techniques one can find the degree of the preferred orientation and construct the so-called pole figure of the grain/crystallite orientations.

If the crystallites are smaller than, say, several hundred nanometres and/or if they are strained and contain point, planar and/or volume defects, their diffraction maxima become broader than the instrumental broadening inherent in the diffractometer itself. This broadening can be noticed first for diffraction maxima at high Bragg angles, where the spectral doublet, $K \alpha_{1} \alpha_{2}$, may gradually cease to be resolved. For even smaller crystallites and severe defects, a measurable broadening of diffraction maxima is also noticed for diffraction maxima at smaller Bragg angles. A successful determination of these microstructural parameters strongly depends on the accurate measurement of profiles of diffraction maxima and on the applied mathematical procedure including a minimization of systematic aberrations. ${ }^{[3,16-23]}$

For a given diffraction maximum (diffraction line), the observed profile, $h(\varepsilon)$, is the convolution of the instrumental profile, $g(\varepsilon)$, and pure diffraction profile, $f(\varepsilon)$ :

$$
h(\varepsilon)=\int f(t) g(\varepsilon-t) \mathrm{d} t .
$$

The variable $\varepsilon$ measures the angular deviation of a point from the true Bragg angle $2 \Theta_{0} ; \varepsilon$ and the auxiliary variable $t$ have the dimension of $2 \Theta$. The profile $f(\varepsilon)$ is caused by small crystallites, strains and other defects in the crystallites, e.g. errors in sequence of crystal lattice planes (stacking faults). ${ }^{[3,16]}$ It can be deduced from the measured profiles, $h(\varepsilon)$ and $g(\varepsilon)$, by the Fourier transform, the socalled Stokes procedure, without any assumptions in the mathematical representation of the measured profiles. According to Warren ${ }^{3}$, the dependence of the Fourier coefficients of the profile $f(\varepsilon)$ on the distance $L$, which is normal to the set of crystal lattice planes corresponding to the 
diffraction maximum, yields the crystallite size and the distribution of the crystallite sizes. Analogously to (4), the pure diffraction profile, $f(\varepsilon)$, is the convolution of the crystallite size/faulting profile, $p(\varepsilon)$, and the strain profile, $s(\varepsilon)$ :

$$
f(\varepsilon)=\int p(t) s(\varepsilon-t) \mathrm{d} t
$$

In order to find the solution of (5), it is necessary to assume mathematical functions for the unknown profiles $p(\varepsilon)$ and $s(\varepsilon)$. If it is assumed that $p(\varepsilon)$ is the Cauchy function and $s(\varepsilon)$ is the Gaussian function (which is often cited in the literature), it follows that for the integral widths of the profiles $f(\varepsilon), p(\varepsilon)$ and $s(\varepsilon)$ the following (approximate) equation is valid:[17,18]

$$
\beta_{i}^{2}=\beta_{i} \beta_{p i}+\beta_{s i}^{2}
$$

The application of (6) to two or more orders of the diffraction maximum corresponding to the same set of crystal lattice planes, yields the crystallite size, in direction normal to the planes, and the strain in the crystallite (e.g. Refs. 3 , and 16).

The Stokes deconvolution followed by the WarrenAverbach and integral widths analyses has been recently implemented in easy-to-use program, XBroad, for a quick determination of the basic microstructural information from X-ray powder diffraction patterns (Skoko et al., J. Appl. Cryst. 2012, 45, 594-597). [23]

\section{CRYSTAL STRUCTURE}

X-ray diffraction and the complementary techniques of electron and neutron diffraction are exceptional and unique methods in determination of the crystal structure of a substance, that is, the parameters of the unit cell, the space periodical arrangement of atoms/ions/molecules in the crystal, identification of particular atoms, determination of their coordinates, nature and length of their mutual chemical bonds, angles between the chemical bonds, symmetry elements which define the space group (e.g. Fundamentals of Crystallography, 1992, ${ }^{[24]}$ Direct Phasing in Crystallography, 1998, [25] Structure Determination from Powder Diffraction Data, 2002,[26] and later editions of these textbooks).

The integrated intensity of a given diffraction maximum $h k l$ is proportional to the power of X-ray radiation diffracted in a narrow solid angle about the direction strictly given by the Bragg law (1); it is defined by several factors:

$$
I_{h k l} \sim F_{h k l}^{2}(\text { PTA })_{h k l} \text {. }
$$

The factor $P$ takes into account the polarization of diffracted rays and the geometry of the diffractometer, the factor $T$ includes the thermal vibrations of atoms and the factor $A$ the absorption of $X$-rays in the specimen. $F_{h k l}$ is the structure factor which contains the number, sort and positions of atoms in the unit cell:

$$
F_{h k l}=F_{h k l} \exp \left(\mathrm{i} \alpha_{h k l}\right)=\sum_{j} f_{j} \exp \left[2 \pi \mathrm{i}\left(h x_{j}+k y_{j}+z_{j}\right)\right] .
$$

The summation is performed over all symmetry independent atoms in the unit cell; $x_{j}, y_{j}$ and $z_{j}$ are the relative coordinates of the $j$-atom in terms of the unit-cell edges, $f_{j}$ is the atomic scattering factor of the $j$-atom, while $\alpha_{h k}$ is the phase of the structure factor. In a simple case (e.g. the substance having the cubic symmetry and a simple chemical composition), the atomic coordinates can be deduced from an appropriate set of the structure factors. By means of the Fourier transform of the structure factors of observed diffraction maxima, the 3D electron density, $\rho(x, y, z)$, in the unit cell, averaged over the whole crystal, can be determined:

$$
\rho(x, y, z) \sim \Sigma_{h} \Sigma_{k} \Sigma_{l} F_{h k l} \exp [-2 \pi \mathrm{i}(h x+k y+l z)] .
$$

Maxima of the electron density function (9) correspond to the atomic positions. However, the coefficients $\boldsymbol{F}_{h k l}$ in (9) are generally complex quantities, having the magnitude and phase, according to (8). One can determine (on a relative scale) the squares of the structure factor, $\boldsymbol{F}_{h k l}^{2}$, from the measured intensity of diffraction maximum, according to (7), but not the phase, $\alpha_{h k l}$. Therefore, (9) does not provide, in a general case, a direct determination of the crystal structure.

The contemporary X-ray diffraction analysis provides several procedures which can be applied in determination of the phases of the structure factors: ${ }^{[24-26]}$

- isomorphous replacement/the heavy atom method: a light atom (having a small atomic number) in the crystal is replaced by a heavy atom (having much bigger atomic number) without a change of the space group; the contribution of the heavy atom to the diffracted intensities is much bigger than that of the replaced atom, this makes it possible to estimate phases of the structure factors and to determine the crystal structure;

- Patterson function: the Fourier transform of the squares of the structure factors, which are proportional to diffracted intensities (7), eliminates the information on the phases of the structure factors; therefore, the maximum of the Patterson function in the point $(U, V, W)$ corresponds to the position vector of one atom in relation to another atom, the position vector having the components $U, V$ and $W$ 
while the maximum in the point $(U, V, W)$ being proportional to the product of the electron densities of the two atoms,

$$
P(U, V, W) \sim \Sigma_{h} \Sigma_{k} \Sigma_{1}\left\{\left|F_{h k l}\right|^{2} \cos [2 \pi(h U+k V+I W)]\right\} ;
$$

- direct methods of estimation/determination of the phases of the structure factors: these methods are based on probability expressions applied to the phases of the structure factors of diffraction maxima having highest intensities; this procedure is supported by the fact that the crystal is composed of discrete atoms and that the electron density exists throughout the crystal; that enables to relate magnitudes and phases of the structure factors which are otherwise independent quantities.

The choice of the procedure(s) which could be applied in determination of the crystal structure depends on the specific characteristics of the substance under study. All the procedures include the use of complex and sophisticated software systems and powerful computers. The first step is to obtain an approximate model of the crystal structure, which is followed by a process of the refinement of all parameters determining the crystal structure. In these steps the model and the refined crystal structure should be in accordance with the known requirements for the chemical composition of the substance under study, as well as to the literature data deposited in the Powder Diffraction File, International Centre for Diffraction Data, ${ }^{[10]}$ in the Cambridge Structural Database, Cambridge Crystallographic Data Centre, or in the Inorganic Crystal Structural Database, produced by Fachinformationszentrum, Karlsruhe and National Institute of Standards and Technology, USA.

The refinement procedure is expressed by the discrepancy factor; that factor is a measure of the difference between the refined model of the crystal structure and the true (but not known) crystal structure; it is equal to the sum of absolute differences between the observed and calculated magnitudes of the structure factors divided by the sum of the observed magnitudes of the structure factors (but, there exist other definitions).

\section{X-ray, Electron and Neutron Diffraction}

$\mathrm{X}$-rays and electrons are scattered by electron clouds of the atoms while neutrons are scattered by atomic nuclei while passing through the substance. By X-ray diffraction it may appear difficult to determine the positions of the light atoms (like hydrogen) in the crystal structure containing also heavy atoms. Neutron diffraction enables to determine the positions of light atoms, for instance, the shift of oxygen in supraconductors and ferroelectrics, or the hydrogen bond in the biological system. In order to decrease the incoherent scattering of neutrons on hydrogen, it can be replaced by deuterium. By neutron diffraction one can determine magnetic moments and their space long-range order on the basis of appearance of additional diffraction maxima.

A great advantage of the electron microscope is that it is possible to obtain the high-resolution microscopy image and the diffraction pattern of the same small part/area of the specimen, e.g. of a precipitate in the (super)saturated solid solution, thus enabling a direct study of the relation of the crystal lattice planes inside the precipitate and in the surrounding matrix, i.e. the orientation of the precipitate in the matrix and the crystal structure of the precipitate. Recent studies of crystalline nanomaterials by electron microscopy and electron diffraction in Zagreb are described in a review paper by A. M. Tonejc, appearing in Crystallography in Croatia, published by Croatian Academy of Sciences and Arts (2012).

\section{RECENT ADVANCEMENT}

Most of the classic techniques and methods for obtaining the diffraction pattern of the polycrystal and their interpretation in terms of information on the crystal structure and microstructure were introduced before the development of contemporary sophisticated instruments and powerful and fast computers. By means of the modern diffractometer and the adequate software it is possible to collect an enormous amount of diffraction data in a short real time. For instance, the derivation of the pure diffraction profile by the Stokes deconvolution from the observed and instrumental profiles of a given diffraction maximum may be performed in several minutes. The use of the synchrotron radiation, the intensity of which is orders-of-magnitude bigger than that of the classic source of X-rays, opens many versatile possibilities in the study of the crystal structure and microstructure, for instance, the resolution of dynamic processes in the crystalline substance which happen in time intervals shorter than a picosecond. Recent trends in the development of diffraction techniques have been described e.g. in monographs Defect and Microstructure Analysis by Diffraction ${ }^{[16]}$ and Structure Determination from Powder Diffraction Data ${ }^{[26]}$ containing papers by prominent scientists in the field.

The introduction of the analytical bell-shaped functions (Gaussian, Cauchy, Voigt and others) in description of the profiles of diffraction maxima has represented a significant advance in interpretation of the diffraction pattern of the polycrystal. The importance of that advance has become obvious in the last decades. That has led to the development of the well-known Rietveld method ${ }^{[27,28]}$ and to the diffraction pattern decomposition techniques, to the separation of the profiles of the overlapping diffraction maxima $^{[20]}$ and to the general concept of the whole powder pattern fitting, i.e. the decomposition of the whole 
diffraction pattern in its components, e.g. ${ }^{[29]}$ The whole powder pattern modelling allows a simultaneous processing of the overall X-ray diffraction pattern; it is based on a suitable model of the crystallite size/shape and strain, which could be applied along the whole diffraction pattern (e.g. Scardi et al. ${ }^{[30]}$ ).

The Rietveld method is a so-called full pattern analysis technique. The assumed model of the crystal structure and microstructure of the studied substance, together with known instrumental parameters of the diffractometer, are used to generate the theoretical diffraction pattern that is compared to the observed pattern. The least squares procedure is used to minimize the difference between the calculated pattern and the observed pattern by adjusting the model parameters in a series of iterative steps. The residual, $S$, which is minimized, is of the form

$$
S=\sum w_{i}\left|y_{i 0}-y_{i c}\right|^{2}
$$

where $y_{i o}$ is the intensity observed at the ith step in the diffraction pattern and $y_{i c}$ the intensity calculated via the structural model at the same step, while $w_{i}$ is a suitable weight. That procedure may result in determination of the crystal structure and microstructure to a satisfactory and credible degree, which is in line with the chemical requirements.

However, the Rietveld method may be rather challenging even for a well-trained crystallographer, due to the overlapping of diffraction maxima (which may be a severe problem for a low-symmetry substance having a big unit cell), possible errors in determination of the Miller indices (especially at high Bragg angles), the presence of the preferred orientation of the crystallites, an inaccurate estimation of the background line etc.

There are several excellent software packages for the interpretation of the diffraction pattern of the polycrystal which are used by the crystallographic community worldwide. One of these systems of programmes is EXPO2013 which has been continuously upgraded by computing and graphical tools, in order to efficiently solve structures in short time and in an (as possible) automatic way. That system contains all the steps which are necessary in determination of the crystal structure and microstructure: indexing of diffraction maxima, definition of the space group, the choice of the model of the crystal structure based on the estimation of the phases of structure factors by application of direct methods in the reciprocal space and the crystal space, the refinement of the model of the crystal structure by application of the Rietveld method and other procedures, etc. ${ }^{[31]}$

The recent development of the determination of the crystal structure by X-ray diffraction in the polycrystal yields the solution of a reliable level in most cases, with hundreds Rietveld refined small-molecule structures published each year. However, unlike X-ray diffraction in the single crystal, the discrepancy factor may be unreliable, ${ }^{[32]}$ and the e.s.d. values of the structural parameters may be misleading. ${ }^{[33]}$ In addition, a typical Rietveld refinement may be restrained, making it difficult to estimate the reliability of the resulting structural parameters. Recently, a method for checking the validity and accuracy of the structure refined by the Rietveld method has been proposed, requiring only to change the individual values and the overall weight of the restraints. ${ }^{[34]}$

Due to recent upgrades of synchrotron- and laboratory sources and the development of position sensitive detectors and reaction cells, it is possible to collect in situ many powder diffraction patterns in dependence on external variables (i.e. temperature and/or pressure) with a time resolution in the far sub-second regime thus allowing to track complex structural changes in detail. The real challenge is to obtain a maximum amount of information from such high quality data. In principle, there is many information contained in the powder diffraction pattern, but much effort is needed to reveal these, often hidden, information. In the last decade, many new ideas have been successfully applied to find ways to reveal such information (e.g. Dinnebier et al. ${ }^{[35]}$ ).

Let us mention several examples of the application of contemporary X-ray difraction techniques from the literature.

The crystal structure of a given substance can be studied as a function of temperature and/or pressure and refined by the Rietveld method, this being an excellent tool for the definition of the phase diagram. A high pressure diffraction beamline at the European Synchrotron Radiation Facility (ESRF) uses the monochromatic radiation with a large area detector. The diffraction experiment with a single crystal and a polycrystal can be performed at high pressure in the diamond anvil cell, permitting accurate determination of the crystal parameters. Recent technical advances have significantly contributed to the utility of Xray diffraction at high pressure. ${ }^{[36]}$ New ways of supporting the diamond anvil have considerably increased the volume of accessible reciprocal space. The use of helium or neon as the pressure transmitting medium extends substantially the practicable pressure range. The data can be collected at low and high temperatures. Also, the single crystal diffraction with laser heating has become possible. ${ }^{[37]}$

In the field of ultrafast $\mathrm{X}$-ray diffraction and related techniques, such as inelastic or diffuse $\mathrm{X}$-ray scattering, the structural motifs in the crystal are excited by ultrashort light pulses and the dynamics on the natural timescale of atomic motion is monitored, with a resolution of about hundred femtoseconds. In the recent experimental progress both laser-based plasma X-ray source and synchrotron 
radiation are used. The inhomogeneous excitation of a metallic thin film and the associated generation of hypersound waves can be monitored. This process is used to tailor giant phonon-wavepackets with a well defined wavevector, although localized to few tens of nanometers. One can not only observe the generation and decay of the phonons but also monitor nonlinear phonon-phonon interaction in the real time. Structural changes in rare earth metals being heated by ultrashort laser pulses can be studied. The transient lattice strain can be used as a local ultrafast thermometer, that shows the heat flow within a multilayered sample, where a very large fraction of the heat can be trapped in antiferromagnetic excitations. Therefore, complex crystal lattice dynamics induced by various sources, fluctuations during the phase transition and crystal lattice relaxation phenomena can be studied. ${ }^{[38]}$

Real-life energy-related materials such as solid-state hydrogen storage compounds or components of electrochemical cells are usually polycrystalline, poorly crystallized, highly reactive and dynamic systems. Powder diffraction obtained by a modern high brilliance $\mathrm{X}$-ray source is the most useful tool to investigate such systems because it is easy, fast and versatile with respect to measurement conditions as well as in situ setups. However, powder diffraction applied to these systems rapidly reaches its limits due to the low crystallinity of samples prepared by mechanochemistry as well as due to the method itself. It can be shown how a complementary approach, combining powder diffraction with non-diffraction methods such as vibrational spectroscopy, thermal analysis and supported by $a b$ initio solid state calculations, allows to overcome some of these limitations. ${ }^{[39]}$

As stated above, the analysis of the X-ray diffraction pattern yields the microstructural parameters, which are usually named as the average crystallite size and (micro)strain; however, their true physical meaning is often ignored. The consequence is that qualitative results are often mistaken as fully quantitative ones. In the past decades, a set of full pattern methods has been proposed to extract quantitative microstructure data from the diffraction pattern by considering physical models for the substance. In that way, a distribution of the crystallite size and shape and strains can be quantified together with other structural data. The recent advance in the field leads to the possibility to extract the quantitative data from the diffraction pattern of the substance that is not fully $3 D$ periodic, extending down to the $2 \mathrm{D}$ periodic case. ${ }^{[40]}$

An important and traditional method for characterization of a polymer/cellulose is X-ray diffraction, which can reveal crystallinity and crystallite size. However, several problems complicate the evaluation of a typical diffraction pattern which may show only few distinct features. Diffraction maxima are usually broad, due to small crystallite (domain) size, this causing their overlapping. The maxima are affected by the preferred orientation effect, due to the fibrous nature of the polymer, and due to a low absorption coefficient, this introducing a shift and asymmetry of diffraction maxima. Anyway, the Rietveld method is preferred in interpretation of the diffraction pattern of a polymer, but the challenge is to minimize the number of refined parameters and thereby to correlate the parameters, in order to deduce a reliable microstructure. ${ }^{[41]}$

Some molecular systems exist near the edge of crystallinity. Often, despite repeated crystallization attempts, these materials, at best, form polycrystalline solids and frequently exhibit substantial defects in crystal growth. A study of two hundred substances with unknown crystal structure, from poorly crystallizing ones to those which easily form single crystals, has been undertaken using the ESFR beamline. The substances have been divided into groups in line with their chemical composition. According to their powder diffraction patterns, some substances have been amorphous, but many substances have shown diffraction data of a sufficient quality that has enabled determination of the crystal structure and the nature of microstructural imperfections, such as small crystallite size, crystal lattice strain and stacking faults. ${ }^{[42]}$

The experiments performed with free electron lasers on small crystals of proteins have opened a new field, the serial crystallography (Chapman et al. 2011). ${ }^{[43]}$ The pulses from X-ray free-electron lasers are much brighter than the brightest synchrotron beams available today. When focused to a micrometre particle, such a pulse destroys any substance, but the pulse terminates before significant atomic motion can take place. This mode of diffraction before destruction yields structural information on proteins (at a resolution of $\sim 0.2 \mathrm{~nm}$ ) that cannot be grown into large enough crystals or are too radiation sensitive for high-resolution crystallography. This has opened a new methodology of serial femtosecond crystallography that yields radiation damage-free structures without the need for cryogenic cooling of the sample. ${ }^{[43]}$

The Rietveld method can be also applied to electron diffraction: the first paper was that of Weirich et al. ${ }^{[44]}$ and the next one by A. M. Tonejc et al. ${ }^{[45]}$

\section{EXAMPLES}

The evidence for statements as well as experimental data on the heating/quenching rates and heating/cooling/milling times in the following studies are given in the corresponding references.

\section{Phase Transitions in Titanium Dioxide}

Titanium dioxide, $\mathrm{TiO}_{2}$, appears in nature, under a standard pressure and temperature, in three crystal polymorphs: anatase (space group $14_{1} /$ amd; unit-cell parameters at $25^{\circ} \mathrm{C}$ : $a=3.784(2), c=9.514(5) \AA$; $Z=4$; density = $\left.3.89 \mathrm{~g} \mathrm{~cm}^{-3}\right)$; rutile $\left(P 4_{2} / \mathrm{mnm}\right.$; unit-cell parameters at $25^{\circ} \mathrm{C}: a=4.594(1)$, 


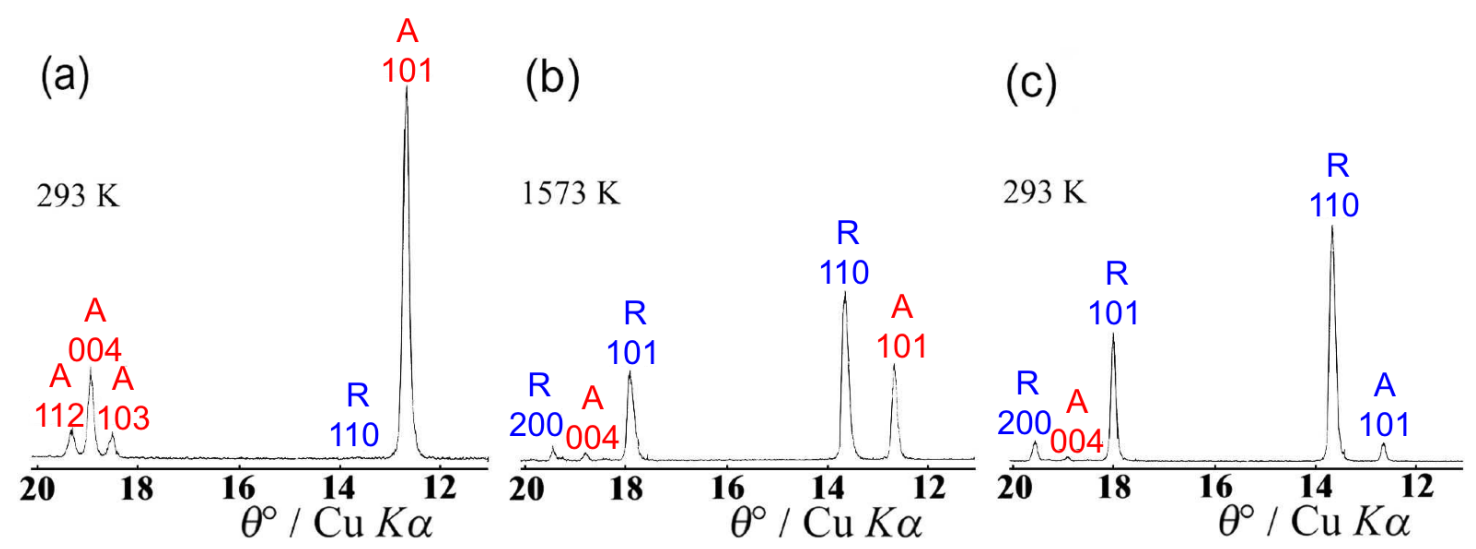

Figure 1. Characteristic parts of XRD patterns of the as-synthesized $\mathrm{TiO}_{2}$ at (a) RT, (b) $1573 \mathrm{~K}$, (c) RT, after a complete heating and cooling cycle; $\mathbf{A}=$ anatase, $\mathbf{R}=$ rutile.

$c=2.958(1) \AA ; Z Z=2$; density $=4.25 \mathrm{~g} \mathrm{~cm}^{-3}$, brookite (space group $\mathrm{Pbca}$; unit-cell parameters at $25^{\circ} \mathrm{C}: a=9.19(2), b=$ 5.46(1), $c=5.15(1) \AA ̊$; $Z=8$; density $\left.=4.12 \mathrm{~g} \mathrm{~cm}^{-3}\right)$. In literature, a high-pressure phase, $\mathrm{TiO}_{2} \mathrm{II}$, has been also described (space group Pbcn; unit-cell parameters at $25^{\circ} \mathrm{C}$ : $a=4.515(6), b=$ 5.497(5), $c=4.939(5) \AA, Z=4$; density $\left.=4.33 \mathrm{~g} \mathrm{~cm}^{-3}\right) \cdot{ }^{[10]}$

The phase transition of anatase, $\mathbf{A}$, to rutile, $\mathbf{R}$, does not take place at a fixed temperature, and the data found in the literature are rather contradictory. The temperature and kinetics of the transition $\mathbf{A} \rightarrow \mathbf{R}$ depend on the characteristics of the starting $\mathbf{A}$, such as particle/crystallite size (specific surface), strains in the crystallites, the content and kind of impurities, deviation from stoichiometry, as well as on the atmosphere and pressure to which the material is exposed. ${ }^{[46]}$

The phase transition $\mathbf{A} \rightarrow \mathbf{R}$ was followed by in situ $\mathbf{X}$ ray diffraction. The experiments were performed with an as-synthesized $\mathrm{TiO}_{2}$ p.a., high-energy ball-milled $\mathrm{TiO}_{2}$ p.a. and the milled and pressed $\mathrm{TiO}_{2}$.

Characteristic parts of XRD patterns of the as-synthesized $\mathrm{TiO}_{2}$ p.a. at RT, at $1573 \mathrm{~K}$ and again at RT, after a complete heating and cooling cycle, are shown in Figure 1. The dominant phase at RT (before heating) was $\mathbf{A}$, while $\mathbf{R}$ was present in traces. One can notice rather sharp diffraction lines due to big crystallites (estimated to 140 to $150 \mathrm{~nm}$ in size). During the heating run, the intensities of diffraction lines of $\mathbf{A}$ slowly decreased due to increased thermal vibrations of atoms. At the same time, diffraction lines shifted toward smaller Bragg angles due to thermal expansion, which was found anisotropic. Above $\approx 1200 \mathrm{~K}$ a gradual transition $\mathbf{A} \rightarrow \mathbf{R}$ took place. The nuclei of $\mathbf{R}$ were formed both at the surface and in the interior of the $\mathbf{A}$ crystallites, and these nuclei grew in number and size. At the highest reached temperature in this experiment, $1573 \mathrm{~K}$, the fraction of $\mathbf{R}$ was bigger than that of $\mathbf{A}$. During the cooling run, the transition $\mathbf{A} \rightarrow \mathbf{R}$ continued and apparently ceased below $\approx 600 \mathrm{~K}$. At RT, after cooling, $\mathbf{R}$ was the dominant phase, with several molar percent of $\mathbf{A}$. $\mathbf{R}$ also exhibited an anisotropy in thermal expansion. The width of diffraction lines of both $\mathbf{A}$ and $\mathbf{R}$ depended very little on temperature during the heating and cooling runs. The dependence of the molar fractions of $\mathbf{R}$ and $\mathbf{A}$ on temperature is shown in Figure 2. In this work it was concluded that the preferred orientation of crystallites in the sample was absent. Then, one can find the molar fractions of the phases by comparison the measured and calculated intensities of diffraction lines, for instance of the $\mathbf{R}$ diffraction line 110 , and of the $\mathbf{A}$ diffraction line 101 , according to $\mathbf{R} / \mathbf{A}=$ $1.25\left(I_{110 R} / I_{101 \mathrm{~A}}\right) \cdot{ }^{[46]}$

The high-energy ball-milling of the as-synthesized $\mathrm{TiO}_{2}$ induced phase transitions $\mathbf{A} \rightarrow \mathbf{R}, \mathbf{A} \rightarrow \mathrm{TiO}_{2}$ II, or $\mathbf{A} \rightarrow \mathrm{TiO}_{2} \mathrm{II} \rightarrow \mathbf{R}$.

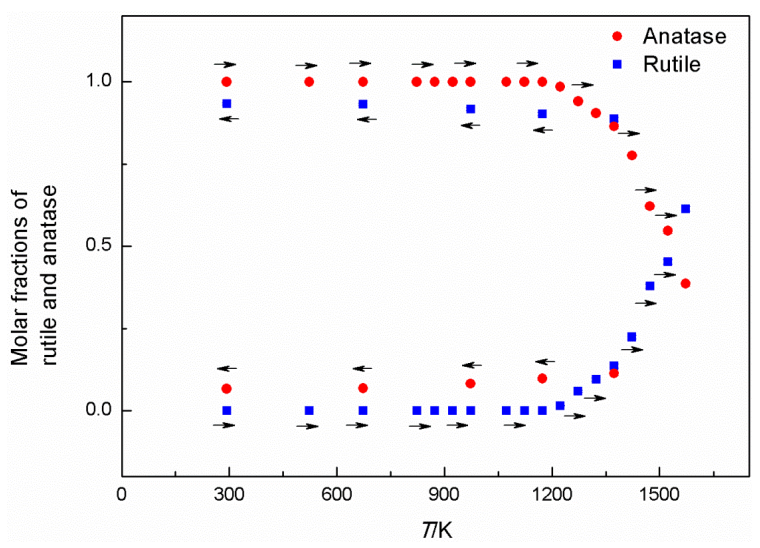

Figure 2. The dependence of molar fractions of $A$ and $R$ on temperature of the as-synthesized $\mathrm{TiO}_{2}$ during a complete heating and cooling cycle. The arrows indicate the sense of the temperature change. 
(a)

$$
293 \mathrm{~K}
$$

(b)

$1373 \mathrm{~K}$

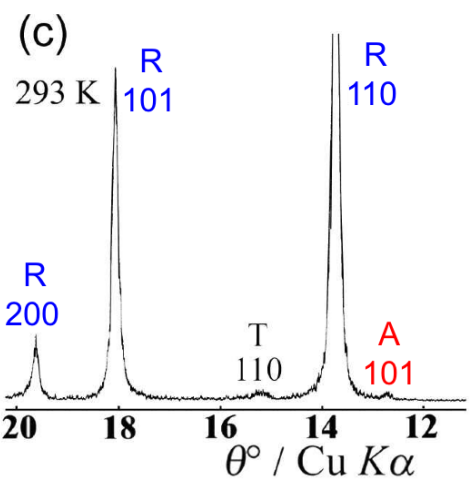

Figure 3. Characteristic parts of XRD patterns of the high-energy ball-milled $\mathrm{TiO}_{2}$ at (a) RT, (b) $1373 \mathrm{~K}$, (c) RT, after a complete heating and cooling cycle; $\mathrm{A}=$ anatase, $\mathrm{R}=$ rutile, $\mathrm{T}=\mathrm{TiO}_{2}$ II.

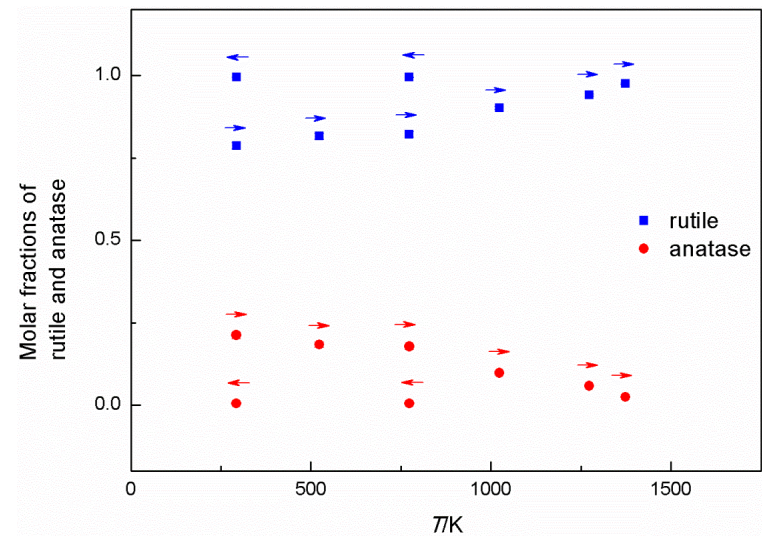

Figure 4. The dependence of molar fractions of $\mathrm{A}$ and $\mathrm{R}$ on temperature of the high-energy ball-milled $\mathrm{TiO}_{2}$ during a complete heating and cooling cycle. The arrows indicate the sense of the temperature change.

The transition $\mathbf{A} \rightarrow \mathbf{R}$ started in the interior of the crystallites, while the transition $\mathbf{A} \rightarrow \mathrm{TiO}_{2} \mathbf{I I}$, and then $\mathrm{TiO}_{2} \mathrm{II} \rightarrow \mathbf{R}$, took place dominantly in their surface layers. The kinetics of these transitions was also followed by Raman spectroscopy, transmission and scanning electron microscopy (TEM, SEM) and by selected-area electron diffraction (SAED). After 10 hours of milling the crystallite size decreased from some $150 \mathrm{~nm}$ to $12(3) \mathrm{nm}$. This fact was evidenced by diffraction line broadening, as well as by TEM and SAED (electron diffraction pattern contained diffraction rings instead of spots). The milling process also introduced strains, which caused an additional broadening of diffraction lines. $\mathbf{R}$ was the dominant phase, the molar fraction of $\mathbf{A}$ was $\approx 0.15$, and that of $\mathrm{TiO}_{2}$ II was $\approx 0.10$. Characteristic parts of diffraction patterns of the milled $\mathrm{TiO}_{2}$ at RT (exhibiting broadened diffraction lines), $1373 \mathrm{~K}$ and again at RT (after a complete heating and cooling cycle) are shown in Figure 3. During heating, a sharpening of diffraction lines was noticed above $\approx 700 \mathrm{~K}$. The transition of $\mathbf{A}$ (the fraction remained after milling) to $\mathbf{R}$ took place soon after the start of heating. At $\approx 1370 \mathrm{~K}$ (the highest temperature in this experiment) the sample contained only small amounts of $\mathbf{A}$ and $\mathrm{TiO}_{2} \mathbf{~ I I}$. In the cooling run to RT the sharpening of diffraction lines went on, due to a continuous increase of the crystallite size and annealing of strains. The fraction of $\mathbf{R}$ continued to increase slowly, while the fractions of $\mathbf{A}$ and $\mathrm{TiO}_{2} \mathbf{l l}$ decreased, to 0.02 to 0.03 . At RT, after a complete heating and cooling cycle, diffraction lines of $\mathbf{R}$ were as sharp as the ones of the non-milled sample. The dependence of the molar fractions of $\mathbf{R}$ and $\mathbf{A}$ on temperature of the milled $\mathrm{TiO}_{2}$ during a complete heating and cooling cycle is shown in Figure 4. The data in this Figure were plotted in terms of $\mathbf{A}+\mathbf{R}=1$, i.e. fraction of $\mathrm{TiO}_{2}$ II was not taken into account. Therefore, the phase transition $\mathbf{A} \rightarrow \mathbf{R}$, initiated by the milling process, was completed by thermal agitation of the sample. ${ }^{[46]}$

The milled and pressed as-synthesized $\mathrm{TiO}_{2}$ powder showed a dependence of the molar fractions of $\mathbf{R}$ and $\mathbf{A}$ on temperature similar to that of the milled sample. An overall thermal behaviour of this sample was more or less similar to that of the milled sample. ${ }^{[46]}$

\section{The Phase Diagram of $\mathrm{Ga}_{2} \mathrm{Se}_{3}-\operatorname{In}_{2} \mathrm{Se}_{3}$}

A series of solid solutions of $\left(\mathrm{Ga}_{x} \mid \mathrm{n}_{1-x}\right)_{2} \mathrm{Se}_{3}, 1 \geq x \geq 0$, were prepared from the melt. In the samples slowly cooled to RT four phases were detected: ${ }^{[47]}$

$1 \geq x \geq 0.88$ : cubic phase $\mathrm{ZB}$, analogous to $\mathrm{Ga}_{2} \mathrm{Se}_{3}$, (zinc blende type), showing asymmetrically broadened diffraction lines, especially those with $h+k+l \neq 4 n$;

$0.88 \geq x \geq 0.75$ : a metastable two-phase field, $Z B+$ hexagonal phase $\gamma_{2}$; the unit cell of $Z B$ increases with the fraction of In;

$0.75 \geq x \geq 0.60$ : one-phase field, $\gamma_{2}$; the unit-cell edges of $\gamma_{2}$ do not change with $x$; 
$0.60 \geq x \geq 0.55$ : two-phase field, $\gamma_{2}+$ hexagonal phase $\gamma_{1} ; \gamma_{1}$ being isostructural with the high temperature phase $\gamma-\ln _{2} \mathrm{Se}_{3}$

$0.55 \geq x \geq 0.02$ : one-phase field, $\gamma_{1}$; the unit-cell edges of $\gamma_{1}$ increase with the fraction of In, while the relative intensities of diffraction lines depend on the ratio $\mathrm{Ga} / \mathrm{In}$, this may indicate at least two cation positions with different occupancy;

$0.02 \geq x \geq 0.005$ : two-phase field, $\gamma_{1}+\alpha$; the phase $\alpha$ being isostructural with the room-temperature phase $\alpha-\ln _{2} \mathrm{Se}_{3}$;

$x=0: \alpha-\ln _{2} \mathrm{Se}_{3}$; the phase transitions $\alpha \rightarrow \beta \rightarrow \gamma \rightarrow \delta$ are detected from room temperature to the melting point; the high-temperature phases can be undercooled to room temperature.

The phase diagram of $\left(\mathrm{Ga}_{x} \mid \mathrm{n}_{1-x}\right)_{2} \mathrm{Se}_{3}$ at RT is shown Figure 5., where $a_{N}$ and $c_{N}$ are normalized unit-cell edges: $\left(a_{N}\right)_{\mathrm{ZB}}=a_{\mathrm{ZB}} / 2^{1 / 2}$ (one half of the face diagonal of the unit cell), $\left(c_{N}\right)_{Z B}=a_{Z B} \times 3^{1 / 2}$ (body diagonal of the unit cell); $\left(a_{N}\right)_{\gamma 1, \gamma 2}=a_{\gamma 1, \gamma 2} / 3^{1 / 2},\left(c_{N}\right)_{\gamma 1, \gamma 2}=c_{\gamma 1, \gamma 2} / 2 ;\left(a_{N}\right)_{\alpha}=a_{\alpha},\left(c_{N}\right)_{\alpha}=$ $c_{\alpha} / 3$.

The thermal expansion of the phase $\gamma_{2}$ is anisotropic but in average comparable to that of the phase ZB. However, the anisotropy in the thermal expansion of the phase $\gamma_{1}$ is very pronounced, being eight times bigger along the $c$ axis than along the $a$-axis. The metastable phase diagram is shown Figure 6(a), which is based on the phases contained in the samples slowly cooled from the melt to RT. On heating the samples above the no-return temperature (above $\approx 1100 \mathrm{~K}$ for $x=0.60$ ), but below the melting point, the phase $\gamma_{2}$ transforms into the phase $Z B$, which shows sharp diffraction lines. On cooling, this phase ZB persists down to $\mathrm{RT}$, and also on repeated heating and cooling cycles, while

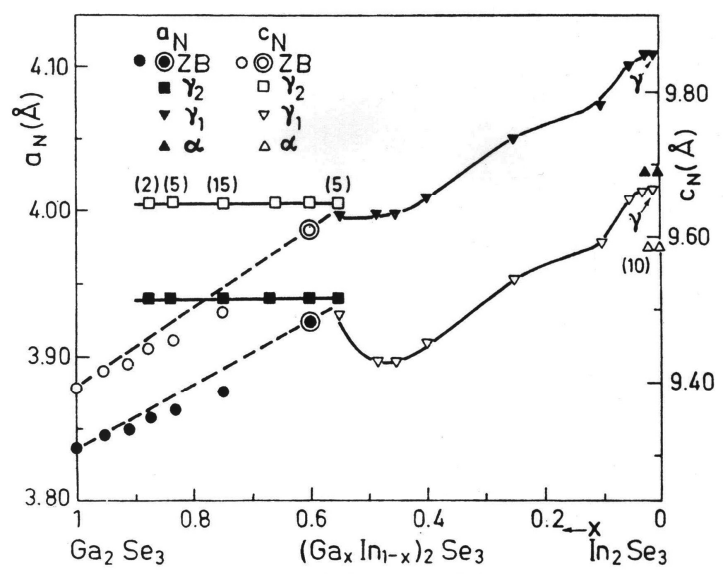

Figure 5. Phase diagram $\left(G a_{x} \mid n_{1-x}\right)_{2} S_{3}$ at room temperature: dependence of normalized unit cell parameters, $a_{N}$ and $c_{N}$, on $x$. Extrapolated values of $\left(a_{N}\right)_{Z B}$ and $\left(c_{N}\right)_{Z B}$, after the transition $\gamma_{2} \rightarrow Z B$, are shown by double circles. In parentheses: molar fraction of minor phases.

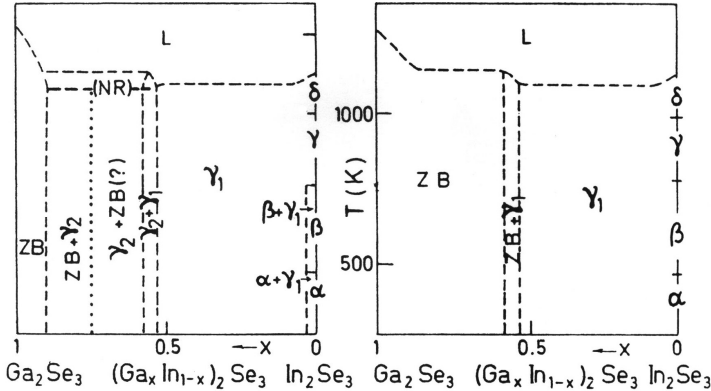

(a)

(b)

Figure 6. Phase diagram $\left(\mathrm{Ga}_{x} \mid \mathrm{n}_{1-x}\right)_{2} \mathrm{Se}_{3}$ : (a) metastable state after the synthesis of samples; $(b)$ equilibrium state, valid after the heating samples above the no return temperature (NR), shown in $(a)$, but below the melting point.

the phase $\gamma_{2}$ does not reappear again. The stable phase diagram is shown in Figure $6(b)$. That diagram shows two wide homogeneous fields, with the phase ZB in the Ga-rich region, and the phase $\gamma_{1}$ in the In-rich region. If the dependence of $\left(a_{N}\right)_{Z \mathrm{ZB}}$ and $\left(c_{N}\right)_{\mathrm{ZB}}$ on $x$ is extrapolated toward $x=0.55$ it follows: $\left(a_{N}\right)_{\mathrm{ZB}} \approx\left(a_{N}\right)_{\gamma 1},\left(c_{N}\right)_{\mathrm{ZB}} \approx\left(c_{N}\right)_{\gamma 1}$ (Figure 5.). ${ }^{[47]}$

\section{Microstructure of Al-Zn Alloys}

A detailed study of Al-Zn alloys, with the molar zinc content, $x(\mathrm{Zn})$, up to 0.62 , by $\mathrm{X}$-ray diffraction and electron microscopy gave many new information on the microstructure of the alloys, in dependence on the initial composition, applied thermal treatment and temperature (Croatica Chemica Acta 2009, 82, 405-420 and other references therein ${ }^{[14]}$.

The alloys rapidly quenched from a temperature, $T_{\mathrm{t}}$, higher than the solid-solution temperature, $T_{s s}$, to RT were supersaturated solid solutions. When aged, at RT or at a higher temperature, these alloys decomposed, and the precipitation of cubic Guinier-Preston zones (GPZ) and hexagonal $\beta$-phase (Figure 7.), as well as the transition of GPZ into the $\beta$-phase took place. During ageing, even at RT, a gradual transition of the cubic $\alpha(\mathrm{M} / \mathrm{GPZ})$-phase, in a metastable equilibrium with $G P Z$, to the cubic $\alpha(M / \beta)$-phase, in an equilibrium with the $\beta$-precipitates, was followed (Figure 8.). The microstructure of the quenched and aged alloys was compared with that of the alloys slowly cooled from $T_{\mathrm{t}}$ to RT (Figure 9.). The quenched alloys, being aged for a prolonged time, as well as the slowly cooled alloys, approached an equilibrium state, in which the $\alpha(\mathrm{M} / \beta)$-phase coexisted with the $\beta$-phase. However, the microstructures of the two groups of alloys were different. The slowly cooled alloys were much closer to the stable equilibrium state, than the quenched and aged alloys. In the latter, the precipitates were not uniformly distributed in the crystal lattice of the $\alpha(\mathrm{M} / \beta)$-phase, and residual strains in the $\alpha(M / \beta)$-phase around the $\beta$-precipitates were present. An important point was that the quenched-in vacancies at RT 


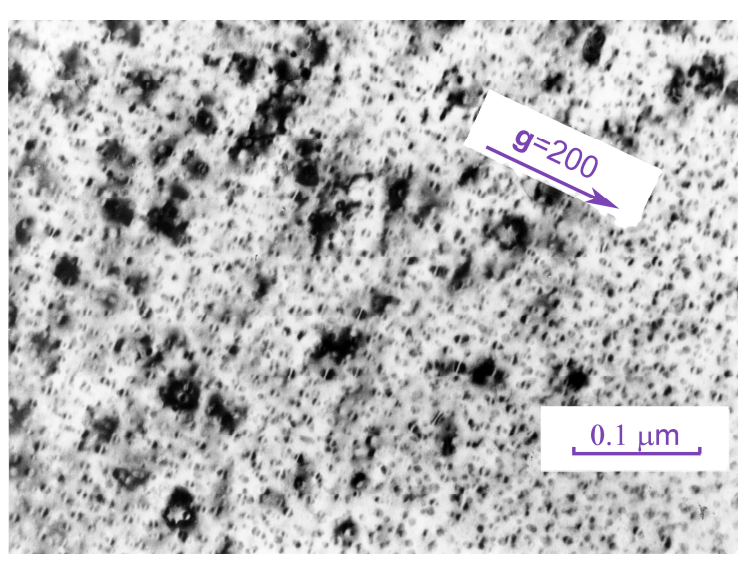

Figure 7. TEM photograph of an Al-rich alloy which was quenched from $T_{\mathrm{t}}$ to RT and aged at $350 \mathrm{~K}$ for 1 day. Initial GPZ and initial $\beta$ precipitates are visible. A line of zero diffraction contrast, normal to the diffraction vector, across the GPZ can be noticed.

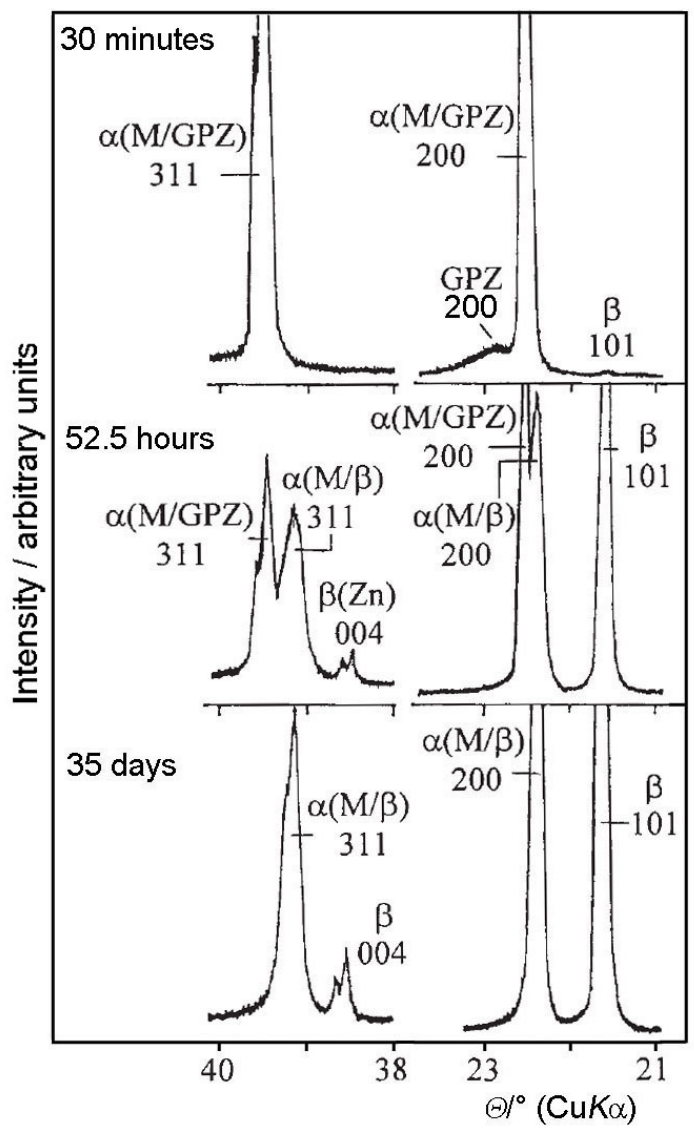

Figure 8. Characteristic diffraction lines of the Al-Zn alloy with $x(\mathrm{Zn})=0.38$. The alloy was quenched from $T_{\mathrm{t}}$ to $\mathrm{RT}$ and aged at RT for 30 minutes, 52.5 hours and for 35 days. A gradual transition from the metastable two-phase system, $\alpha(M / G P Z)+G P Z$, into a more stable two-phase system, $\alpha(M / \beta)+\beta$, can be noticed.
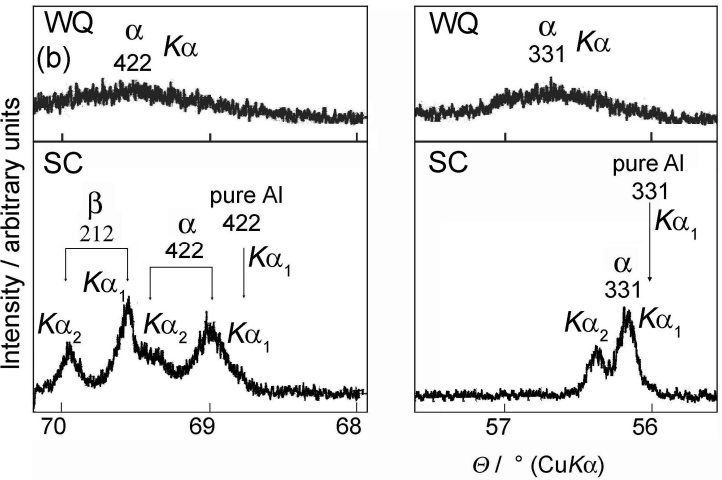

Figure 9. Characteristic diffraction lines of the quenched (WQ) and slowly cooled (SC) Al-Zn alloy with $x(\mathrm{Zn})=0.48$; diffraction pattern of the alloy quenched from $T_{\mathrm{t}}$ to RT was scanned within 30 minutes after quenching.

were much more numerous in the quenched alloys than in the slowly cooled alloys. ${ }^{[14]}$

Both quenched and aged alloys and slowly cooled alloys were gradually heated from RT to $T_{\mathrm{t}}$, and their microstructure was followed in situ by X-ray diffraction; for instance, enhanced thermal vibrations of atoms, thermal expansion, which was found anisotropic for the $\beta$-phase,

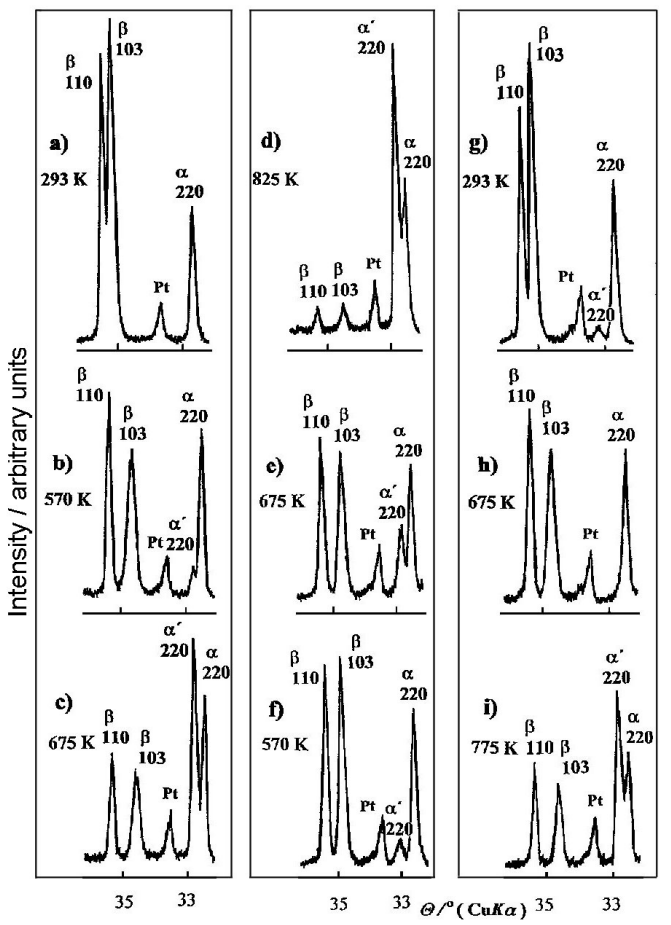

Figure 10. Characteristic diffraction lines of the Al-Zn alloy with $x(\mathrm{Zn})=0.48$, at selected temperatures, including both the first and second heating and cooling cycles. Prior to diffraction study at high temperature, the alloy was quenched from $T_{t}$ to RT and aged at RT for one week. 


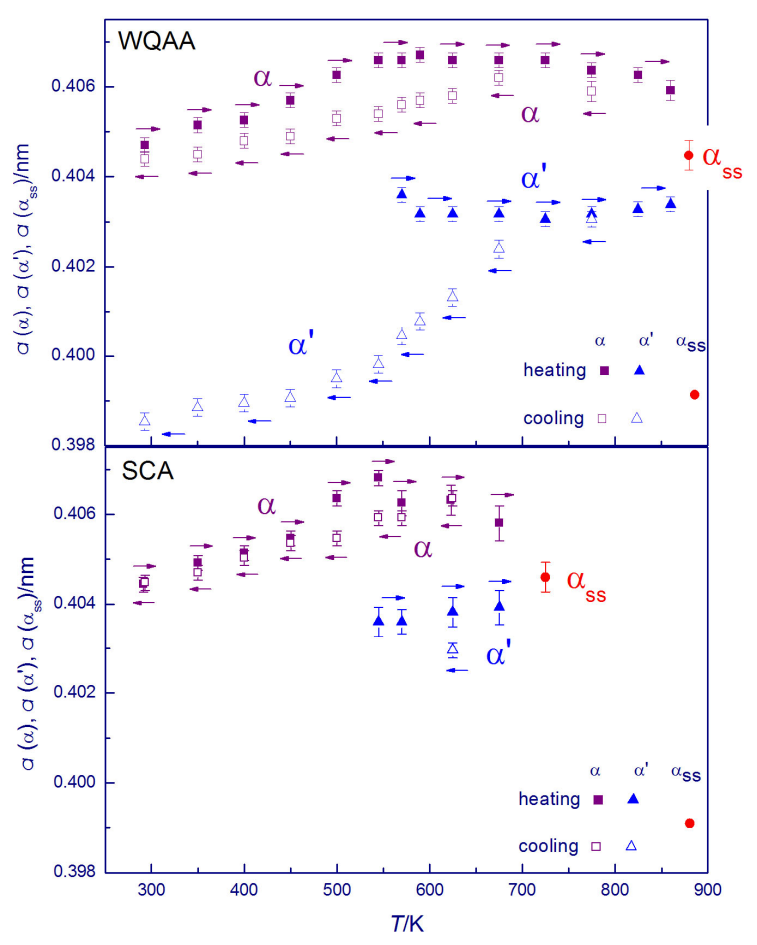

Figure 11. Temperature dependence of the unit-cell parameter $a$ of the phases $\alpha, \alpha^{\prime}$ and $\alpha_{s s}$ in the Al-Zn alloy with $x(Z n)=0.48$ during the first heating and cooling cycle. The upper part is related to the alloy that was quenched from $T_{\mathrm{t}}$ to RT and aged for one week at RT prior to diffraction study at high temperature. The lower part is related to the alloy that was slowly cooled from $T_{\mathrm{t}}$ to RT prior to diffraction study at high temperature. Arrows indicate the sense of temperature change, and vertical bars estimated standard deviation in $a$.

partial dissolution of the $\beta$-phase into the $\alpha(\mathrm{M} / \beta)$-phase, a change of the shape of the $\beta$-precipitates, phase transition of the $\beta$-phase into the cubic $\alpha^{\prime}$-phase, or the transition of both $\beta$-and $\alpha$-phase into the $\alpha^{\prime}$-phase, coexistence of the $\alpha^{\prime}$ - and $\alpha$-phases, or of the $\alpha^{\prime}-, \beta$ - and $\alpha$-phases, a change of composition of particular phases, formation of solid solution, $\alpha_{s s}$ (Figure 10.). $T_{\text {ss }}$ depended on the alloy composition as well as on the thermal treatment.

The behaviour of the quenched and aged alloys during the temperature change was different from that of the slowly cooled alloys, due to their different starting microstructures at RT, prior to examination by X-ray diffraction at high temperature (Figure 11.). Also, the alloys with $x(\mathrm{Zn})$ $\leq 0.40$ behaved at high temperature differently than the alloys with $x(\mathrm{Zn}) \geq 0.44$, the behaviour of the latter not being in line with the phase diagram , as accepted in the literature.

On cooling the alloys from $T_{\mathrm{t}}$ to $\mathrm{RT}$, a temperature hysteresis was observed in reversal phase transitions; that was also proved by DSC. As the heating and cooling runs were performed slowly, most strains in the quenched and aged alloys were annealed, and the resulting microstructure at RT was different from the starting microstructure at $\mathrm{RT}$, concerning the size and shape of the $\beta$-precipitates, their distribution in the crystallites of the $\alpha(\mathrm{M} / \beta)$-phase, and the strains around the precipitates. During the repeated, second, heating and cooling runs, the alloys behaved similarly as they did during the first cooling run but a delay of phase transitions was observed in some cases. An ideal equilibrium state cannot be reached either by slow cooling from $T_{\mathrm{t}}$ to $\mathrm{RT}$, or by prolonged ageing of RT of the rapidly quenched alloys.

This study has confirmed the fact, that the quenched-in vacancies, vacancy-Zn pairs and other vacancy complexes play a dominant role in the diffusion rate of $\mathrm{Zn}$ atoms. In case the alloy is rapidly quenched $\left(\approx 10^{5} \mathrm{~K} / \mathrm{s}\right)$ from a temperature, higher than the solid-solution temperature, to RT, an excess of vacancies is available for the diffusion of $\mathrm{Zn}$ atoms. On the other hand, defects in the crystallites of the $\alpha$-phase, such as dislocations, crystallite boundaries, or incoherent parts of the precipitate $-\alpha$-phase interface, act as sinks (traps) for vacancies. The precipitation processes, i.e. the decomposition of the supersaturated solid solution, depend on the balance of the quenched-in vacancies and traps for vacancies in a particular alloy, subjected to a given thermal treatment. ${ }^{[14]}$

\section{$-\mathrm{Al}_{2} \mathrm{O}_{3}-\mathrm{Fe}_{2} \mathrm{O}_{3}$}

$\alpha-\mathrm{Al}_{2} \mathrm{O}_{3}$ (mineral: corundum) and $\alpha-\mathrm{Fe}_{2} \mathrm{O}_{3}$ (mineral: hematite) belong to the same space group, $R \overline{3} c$ (no. 167). The unit-cell edges at $25^{\circ} \mathrm{C}$, in terms of hexagonal axes are: $a=$ 4.758 and $c=12.991 \AA$ for $\alpha-\mathrm{Al}_{2} \mathrm{O}_{3} ; a=5.034$ and $c=13.752 \AA$ for $\alpha-\mathrm{Fe}_{2} \mathrm{O}_{3}$. The differences in $a$ and $c$ edges are a consequence of different ionic radii of the metals (for coordination six): $0.54 \AA$ for $\mathrm{Al}^{3+}$ and $0.65 \AA$ for $\mathrm{Fe}^{3+}$.

A series of mixtures, having different molar fractions of $\gamma$-AlOOH and $\alpha-\mathrm{Fe}_{2} \mathrm{O}_{3}$ of analytical purity, were mechanically mixed and heated at $1500 \mathrm{~K}$. After heating, the samples were slowly cooled in the furnace to RT. The samples were studied by X-ray diffraction, Mössbauer spectroscopy, field emission scanning electron microscopy (FE-SEM) and energy dispersive X-ray spectrometry (EDS). ${ }^{[48]}$

Two isostructural solid solutions, $A$ and $F$, of the type $\alpha-\left(\mathrm{Al}_{x} \mathrm{Fe}_{1-x}\right)_{2} \mathrm{O}_{3}$ (space group $R \overline{3} c$ ) were formed. The solid solution $\mathrm{F}$, closely related to $\alpha-\mathrm{Fe}_{2} \mathrm{O}_{3}$, was present for $0 \leq x$ $\leq 0.91$, while the solid solution $\mathrm{A}$, closely related to $\alpha-\mathrm{Al}_{2} \mathrm{O}_{3}$, was present for $0.27 \leq x \leq 1$. In the two single-phase intervals, the unit-cell edges $a$ and $c$ of both phases, $F$ and A, decreased as $x$ increased. In the two-phase interval, $0.27 \leq$ $x \leq 0.91$, the unit-cell edges $a$ and $c$ of both phases did not change, whereas the fraction of the phase $F$ decreased and 


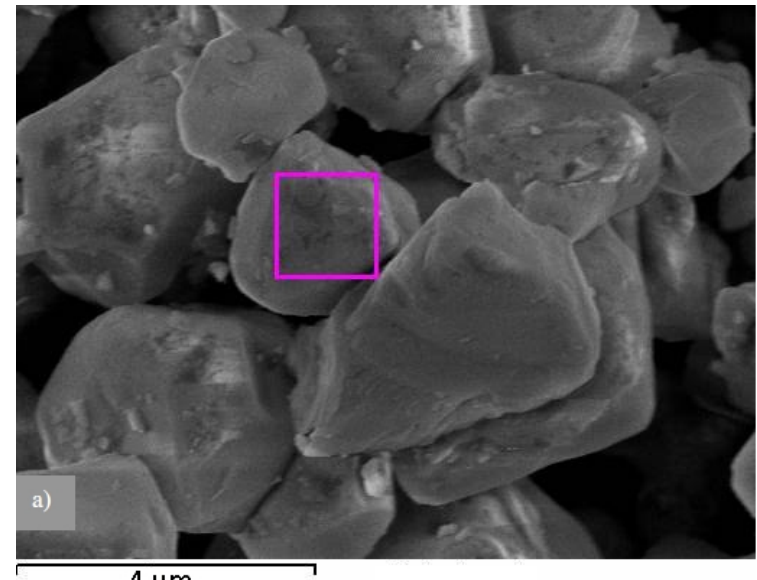

$4 \mu \mathrm{m}$

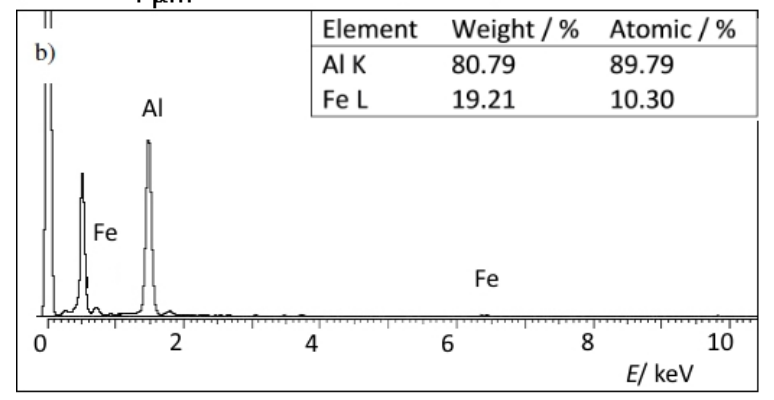

Figure 12. FE-SEM and EDS images of the phase $F$ with $x=0.90$.

the fraction of the phase $A$ increased as $x$ increased. All the samples exhibited sharp X-ray diffraction lines of both phases, $A$ and $F$, with well-resolved spectral doublets

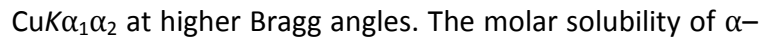
$\mathrm{Al}_{2} \mathrm{O}_{3}$ in $\alpha-\mathrm{Fe}_{2} \mathrm{O}_{3}$ was 0.27 (the phase $\mathrm{F}$ ) and the one of $\alpha-$ $\mathrm{Fe}_{2} \mathrm{O}_{3}$ in $\alpha-\mathrm{Al}_{2} \mathrm{O}_{3}$ was 0.09 (the phase $\mathrm{A}$ ), as calculated from the dependence of the diffraction line intensities of both phases, $\mathrm{A}$ and $\mathrm{F}$, on $x$ by extrapolation to zero intensity. A higher solubility of $\alpha-\mathrm{Al}_{2} \mathrm{O}_{3}$ into $\alpha-\mathrm{Fe}_{2} \mathrm{O}_{3}$ may be due to the fact that the cation $\mathrm{Al}^{3+}$ is smaller than $\mathrm{Fe}^{3+}$. The morphology of crystalline grains and the elemental analysis for the solid solution with $x=0.90$ is shown in Figure 12. ${ }^{[48]}$

\section{Thermosalient Crystals}

Thermosalient crystals, or colloquially commonly called jumping crystals, are potential materials for conversion of thermal or light energy into mechanical energy at the nanometre scale. If a thermosalient crystal is heated or cooled, a sudden and sharp phase transition takes place in it. That transition usually happens in a time interval of several microseconds. During that transition, the crystal experiences a significant discontinuous change in its shape and volume, i.e. in the size of its unit cell, which could be up to $12 \%$. Due to this change the crystal jumps up to a height that is several times its size.
One of the coauthors (ŽS) studied the thermosalient effect in oxitropium bromide by $\mathrm{X}$-ray diffraction, electron microscopy, spectroscopic and thermoanalytical techniques, in order to reveal the mechanism of the jumping phenomenon. ${ }^{[49]}$ Direct observation of the phenomenon and determination of the crystal structure revealed that the jumping of the crystal is a macroscopic manifestation of a highly anisotropic change in the unit-cell volume. The cell distortion is accompanied by a conformational change of the oxitropium cation, which triggers an increased separation among the ion pairs at nearly identical separation between the cation and the anion within each ion pair. That study suggested a mechanism in which a large, thermally accumulated, strain is transferred over the ester bridge to the phenyl ring, which rotates to trigger the phase transition. The high-temperature phase can also be prepared by grinding or UV irradiation of the room-temperature phase. ${ }^{[49]}$

It was reported that N'-2-propylidene-4-hydroxybenzohydrazide showed a behaviour similar to that of the jumping crystal. This system was found in three polymorphic modifications, I, II and III, all having the same polar space group $P n a 2_{1}$, Two phase transitions were observed, I to II and III to II, reported as topotactic (single crystal to single crystal). It was also reported that during the irreversible phase transition from I to II, the polar axis undergoes a strong compression (approximately $15 \%$ ) and a single crystal of the phase $I$ is violently shuttered into single crystal fragments of the phase II (without jumping), while in the reversible phase transition III to II the polar axis expands (approximately $14 \%$ ) and the integrity of the single crystals is preserved - and no movement of crystals was observed. ${ }^{[50]}$

Our recent study (Figures 13. and 14.) showed a different behaviour. ${ }^{[51]}$ During the irreversible phase transition from I to II some of the crystals did indeed shutter into smaller fragments, but a large number remained intact and showed a typical jumping behaviour ${ }^{[49]}$ - jumping all around over large distances (several $\mathrm{cm}$ ). This is typical for other thermosalient materials. Also, during the reversible phase transition, II $\leftrightarrow$ III (both ways), crystals exhibited a jumping behaviour, although somewhat weaker than during the phase transition I to II. This is in contrast to previously reported behaviour ${ }^{[50]}$ and the new model for jumping crystal phenomenon in this system has to be proposed. ${ }^{[51]} \mathrm{A}$ possible explanation of the thermosalient phenomenon may lie in the fact that a change of mutual positions of the molecules due to the change of temperature induced a severe stress in the crystal. In the moment when the stress reaches its maximum, its relaxation takes place; this is manifested in a sharp change of the unit-cell volume and a consequent jumping of the crystal.. ${ }^{[51]}$ 


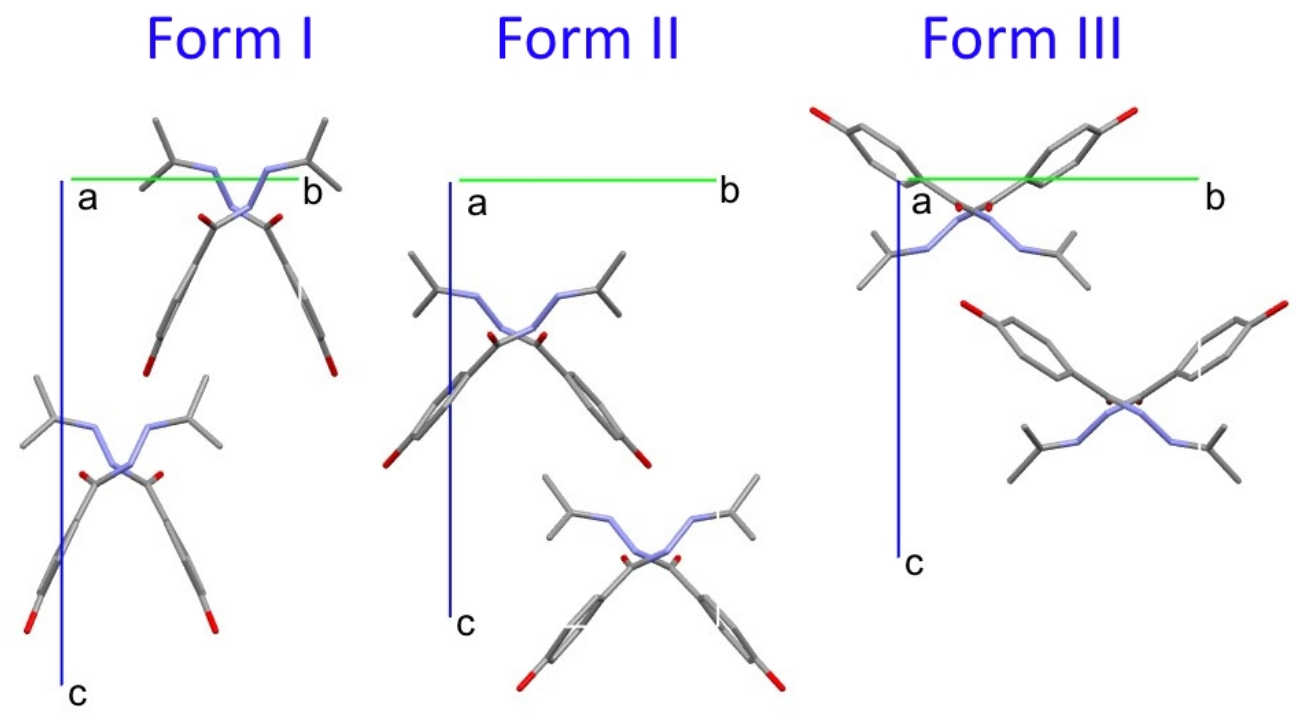

Figure 13. N'-2-propylidene-4-hydroxybenzohydrazide, forms I, II, III.

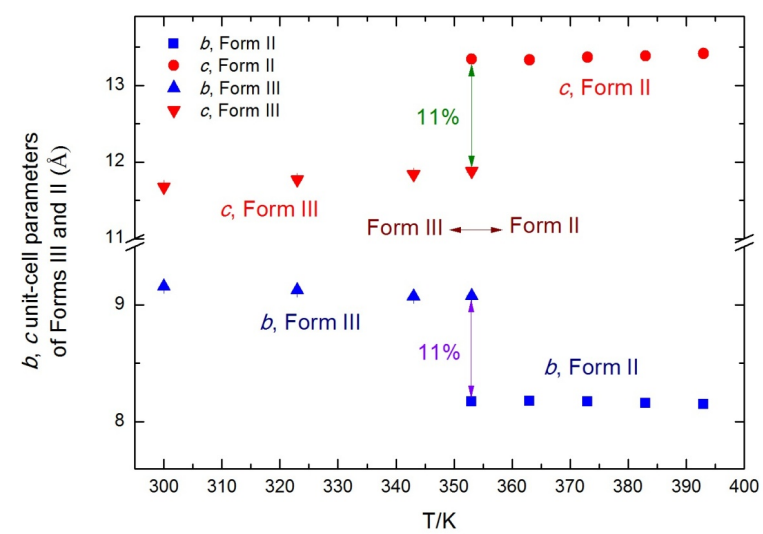

Figure 14. N'-2-propylidene-4-hydroxybenzohydrazide, phase transition $\|\rightarrow\|$, change of unit-cell dimensions.

\section{CONCLUSION}

A crystalline substance is not always available as a single crystal having quality and/or size suitable to be studied by single crystal diffraction techniques. However, many substances appear in the form of a polycrystal or a fine-grained powder. In such a case, X-ray diffraction technique in the polycrystal/crystalline powder is the most appropriate for determination of the crystal structure and microstructure. The interpretation of the powder diffraction pattern is not an easy task. The main difficulties appear due to the overlapping of diffraction lines, background level estimation and preferred orientation of crystallites, which influence the success in determination of the structural data.
Contemporary X-ray, electron and neutron diffraction techniques for study of polycrystalline substances, in synergy with microscopic, spectrometric and other physical techniques, reveal a full insight in crystal structure and microstructure: identification of chemical elements and compounds; elemental analysis; qualitative and quantitative phase analysis; distinction between amorphous and crystalline substances; the amorphous fraction in the multi-phase substance; the local 3D distribution of electron density in amorphous substances (e.g. in metallic glasses); accurate measurement of periodicities in the crystalline substance - unit-cell parameters; solubility limits in solid solutions; precipitation processes in supersaturated solid solutions (e.g. metallic alloys); thermal expansion; phase transitions; phase diagrams; chemical stability and decomposition of chemical compounds; crystallite size, kinetics of crystallite growth, recrystallization, strains, annealing of defects; time resolution of dynamic processes in the crystalline substance; crystal structure, 3D positions of atoms, lengths and nature of chemical bonds, coordination, conformation; resolution of lattice planes and rows of atoms in the crystal, manipulation with particular atoms/molecules; influence of crystal structure, microstructure and defects on physical and chemical properties and on biological activity of a substance, applications in technology and in various field of human activities.

\section{REFERENCES}

[1] H. P. Klug, L. E. Alexander, X-ray Diffraction Procedures for Polycrystalline and Amorphous Materials, John Wiley \& Sons, 1974. 
[2] B. D. Cullity, Elements of X-ray Diffraction, AddisonWesley, Massachusetts, 1978.

[3] B. E. Warren, X-ray Diffraction, Addison-Wesley, Reading MA/Dover, Mineola NY, 1969/1990.

[4] Powder Diffraction - Theory and Practice (Eds.: R. E. Dinnebier and S. J. L. Billinge), Cambridge RSC Publishing, 2008.

[5] P. Debye, P. Scherrer, Phys. Z. 1916, 17, 277.

[6] A. W. Hull, Phys. Rev. 1917, 10, 661.

[7] S. Nishikawa in Fifty Years of $X$-ray Diffration (Ed.: P. P. Ewald), International Union of Crystallography, 1962.

[8] Fifty Years of X-ray Diffraction, International Union of Crystallography (Ed.: P. P. Ewald), A. Oosthoek, Utrecht, 1962.

[9] W. L. Bragg, Proc. R. Soc. Lond. A 1913, 89, 248.

[10] Powder Diffraction File, International Centre for Diffraction Data, Swarthmore, USA; T. G. Fawcett, S. N. Kabekkodu, J. R. Blanton, C. E. Crowder, T. N. Blanton, 29th European Crystallograpic Meeting, Rovinj, 2015, ECA, Book of Abstracts, p. 509; Acta Cryst. A 2015, 71, s511.

[11] F. H. Chung, J. Appl. Cryst. 1974, 7, 519.; F. H. Chung, J. Appl. Cryst. 1974, 7, 526.; F. H. Chung, J. Appl. Cryst. 1975, 8, 17.

[12] S. Popović, B. Gržeta-Plenković, J. Appl. Cryst. 1979, 12, 205.; S. Popović, B. Gržeta-Plenković, T. BalićŽunić, J. Appl. Cryst. 1983, 16, 505.; S. Popović, B. Gržeta-Plenković in Advanced Methods in X-ray and Neutron Structure Analysis in Materials Science (Ed.: J. Hašek), New York, Plenum Press, 1989, 29.

[13] A. Tonejc, D. Ročak, A. Bonefačić, Acta Metall. 1971, 19, 311.; A. Tonejc, J. Mater. Sci. 1972, 1272.; A. M. Tonejc, A. Tonejc, A. Bonefačić, J. Mater. Sci. 1974, 9, 523.; A. Tonejc, A. M. Tonejc, A. Bonefačić, Phys. Lett. 1974, 49A, 145.; S. Popović, A. Tonejc, B. Gržeta, B. Čelustka, R. Trojko, J. Appl. Cryst. 1979, 12, 416.

[14] Ž. Skoko, S. Popović, G. Štefanić, Croat. Chem. Acta 2009, 82, 405.; S. Popović, D. E. Passoja, J. Appl. Cryst. 1971, 4, 427.; S. Popović, H. Löffler, B. Gržeta, G. Wendrock, P. Czurratis, Phys. Status Solidi A 1989, 111, 417.; S. Popović, B. Gržeta, V. Ilakovac, R. Kroggel, G. Wendrock, H. Löfller, Phys. Status Solidi A 1992, 130, 273.; S. Popović, B. Gržeta, H. Löffler, G. Wendrock, Phys. Status Solidi A 1993, 140, 341.; S. Popović, B. Gržeta, H. Löffler, G. Wendrock in Structure and Structure Development in Al-Zn Alloys (Ed.: H. Löffler), Akademie Verlag, Berlin, 1995, 212241.; S. Popović, Ž. Skoko, G. Štefanić, Acta Chim. Slov. 2008, 55, 793.; S. Popović, Ž. Skoko, G. Štefanić, Z. Kristallogr. Suppl. 2009, 30, 309.
[15] S. Popović, J. Appl. Cryst. 1973, 6, 122.; S. Popović, J. Appl. Cryst. 1974, 7, 291.; S. Popović, M. Šljukić, F. Hanic, Phys. Status Solidi A 1974, 23, 265.

[16] Defect and Microstructure Analysis by Diffraction, IUCr Monographs on Crystallography (Eds.: R. L. Snyder, J. Fiala and H. J. Bunge), Oxford University Press, 1999.

[17] F. R. L. Schoening, Acta Cryst. 1965, 18, 975.

[18] N. C. Halder, C. N. J. Wagner, Acta Cryst. 1966, 20, 312.

[19] R. Delhez, Th. de Keijser and E. J. Mittemeijer in Accuracy in Powder Diffraction, NBS Special Publication No. 567 (Eds.: S. Block and C. R. Hubbard), Washington DC, National Bureau of Standards, 1980, pp. 213-253.

[20] J. I. Langford, D. Louër, Rep. Prog. Phys. 1996, 59, 131.

[21] S. Popović, Croat. Chem. Acta 1984, 57, 749.; D. Balzar, S. Popović, J. Appl. Cryst. 1996, 29, 16.

[22] D. Balzar, N. Audebrand, M. Daymond, A. Fitch, A. Hewat, J. I. Langford, A. Le Bail, D. Louër, O. Masson, C. N. McCowan, N. C. Popa, P. W. Stephens, B. Toby, J. Appl. Cryst. 2004, 37, 911.

[23] S. Popović, Ž. Skoko, G. Štefanić, Z. Kristallogr. Proc. 2011, 1, 55.; Ž. Skoko, J. Popović, K. Dekanić, V. Kolbas, S. Popović, J. Appl. Cryst. 2012, 45, 594.; Ž. Skoko, S. Popović, G. Štefanić, J. Mater. Sci. Eng. A 2013, 3, 690.; S. Popović, Ž. Skoko, Maced. J. Chem. Chem. Eng. 2015, 34, 39.

[24] Fundamentals of Crystallography, IUCr Monographs on Crystallography (Eds.: C. Giacovazzo et al.), Oxford University Press, 1992.

[25] Direct Phasing in Crystallography, IUCr Monographs on Crystallography (Ed.: C. Giacovazzo), Oxford University Press, 1998.

[26] Structure Determination from Powder Diffraction Data, IUCr Monographs on Crystallography (Eds.: W. I. F. David, K. Shankland, L. B. McCusker and Ch. Baerlocher), Oxford Science Publ., 2002.

[27] H. M. Rietveld, J. Appl. Cryst. 1969, 2, 65.

[28] The Rietveld Method, IUCr Monographs on Crystallography (Ed.: R. A. Young), Oxford University Press, 1993.

[29] P. Scardi in Defect and Microstructure Analysis by Diffraction, IUCr Monographs on Crystallography (Eds.: R. L. Snyder, J. Fiala and H. J. Bunge), Oxford University Press, 1999, pp. 570-596.

[30] P. Scardi, M. Leoni and Y. H. Dong, IUCr Commission on Powder Diffraction, Newsletter 2000, 24, 23.

[31] A. Altomare, C. Cuocci, C. Giacovazzo, A. Moliterni, R. Rizzi, R. Corriero, A. Falcicchio, J. Appl. Cryst. 2013, 46, 1231. 
[32] C. Buchsbaum, M.U. Schmidt, Acta Cryst. B 2007, 63, 926.

[33] R. J. Hill, I. C. Madsen, J. Appl. Cryst. 1986, 19, 10.

[34] I. S. Bushmarinov, A. O. Dmitrienko, A. A. Korlyukov, M. Yu. Antipin, J. Appl. Cryst. 2012, 45, 1187.

[35] T. Friščić, I. Halasz, P. J. Beldon, A. M. Belenguer, F. Adams, S. A. J. Kimber, V. Honkimäki, R. E. Dinnebier, Nature Chem. 2013, 5, 66.; O. V. Magdysyuk, M. Müller, R. E. Dinnebier, C. Lipp, T. Schleid, J. Appl. Cryst. 2014, 47, 701.; M. K. Panda, T. Runčevski, S. Chandra Sahoo, A. A., Belik, N. K. Nath, R. E. Dinnebier, P. Naumov, Nat. Commun. 2014, 5, 4811.; R. E. Dinnebier, 29th European Crystallograpic Meeting, Rovinj, 2015, ECA, Book of Abstracts, p. 68; Acta Cryst. A 2015, 71, s68.

[36] M. Merlini, M. Hanfland, High Pressure Res. 2013, 33, 511.

[37] M. Hanfland, 29th European Crystallograpic Meeting, Rovinj, 2015, ECA, Book of Abstracts, p. 3; Acta Cryst. A 2015, 71, s3.

[38] M. Bargheer, 29th European Crystallograpic Meeting, Rovinj, 2015, ECA, Book of Abstracts, p. 11; Acta Cryst. A 2015, 71, s11.

[39] P. Schouwink, R. Černý, Chimia 2014, 68, 38.; R. Černý, P. Schouwink, Y. Sadikin, M. Brighi, E. Didelot, 29th European Crystallograpic Meeting, Rovinj, 2015, ECA, Book of Abstracts, pp. 52-53; Acta Cryst. A 2015, 71, s51-s52.

[40] M. Leoni, 29th European Crystallograpic Meeting, Rovinj, 2015, ECA, Book of Abstracts, p. 361; Acta Cryst. A 2015, 71, s377.; P. Scardi, M. Leoni, Acta Cryst. A 2002, 58, 190.; M. Leoni, A. F. Gualtieri, N. Roveri, J. Appl. Cryst. 2004, 37, 166.
[41] A. Thygesen, J. Oddershede, H. Lilholt, A. B. Thomsen, K. Stahl, Cellulose 2005, 12, 563.

[42] J. G. P. Wicker, M. Pillong, T. Wagner, W. I. F. David, R. I. Cooper, 29th European Crystallograpic Meeting, Rovinj, 2015, ECA, Book of Abstracts, p. 489; Acta Cryst. A 2015, 71, s511-s512.

[43] H. N. Chapman et al., Nature 2011, 470, 73.; H. N. Chapman, 29th European Crystallograpic Meeting, Rovinj, 2015, ECA, Book of Abstracts, p. 1; Acta Cryst. A 2015, 71, s1.

[44] T. E. Weirich, M. Winterer, S. Seifried, H. Hahn, H. Feuss, Ultramicroscopy 2000, 81, 263.

[45] A. M. Tonejc, I. Djerdj, A.Tonejc, Mater. Sci. Eng. C 2002, 19, 85.

[46] A. Gajović, K. Furić, N. Tomašić, S. Popović, Ž. Skoko, S. Musić, J. Alloys Compd. 2005, 398, 188.; S. Popović, Ž. Skoko, A. Gajović, K. Furić, S. Musić, Fizika A 2005, 14, 19.

[47] A. Tonejc, S. Popović, Gržeta-Plenković, J. Appl. Cryst. 1980, 13, 24.; S. Popović, A. Tonejc, B. Gržeta-Plenković, B. Čelustka, R. Trojko, J. Appl. Cryst. 1979, 12, 416.

[48] M. Ristić, S. Popović, S. Musić, Croat. Chem. Acta 2009, 82, 397.; S. Popović, M. Ristić, S. Musić, Mater. Lett. 1995, 23, 139.

[49] Ž. Skoko, S. Zamir, P. Naumov, J. Bernstein, J. Am. Chem. Soc. 2010, 132, 14191.

[50] R. Centore, M. Jazbinsek, A. Tuzi, A. Roviello, A. Capobianco, A. Peluso, CrystEngComm 2012, 14, 2645.

[51] Ž. Skoko, D. Popović, J. Popović, K. Molčanov, S. Popović, 23rd Slovenian-Croatian Crystallographic Meeting, Logarska dolina, Slovenia, Book of Abstracts, Faculty of Chemistry and Chemical Technology, Ljubljana, 2014, p. 32. 